\title{
On some conjectures and results for the Riemann zeta-function and Hecke series
}

\author{
by
}

\author{
Aleksandar Ivić (Beograd)
}

1. Spectral theory and the function $\mathcal{Z}_{2}(s)$. The spectral theory of the hyperbolic Laplacian has become increasingly important in the theory of the Riemann zeta-function $\zeta(s)$, especially in problems connected with the fourth moment of $|\zeta(1 / 2+i x)|$. For a comprehensive account of this subject we refer the reader to Y. Motohashi's monograph [15]. In this section we shall briefly state some necessary facts from spectral theory and introduce the function $\mathcal{Z}_{2}(s)$, closely related to $|\zeta(1 / 2+i x)|^{4}$.

Let $\left\{\lambda_{j}=\kappa_{j}^{2}+1 / 4\right\} \cup\{0\}$ be the discrete spectrum of the hyperbolic Laplacian

$$
\Delta=-y^{2}\left(\left(\frac{\partial}{\partial x}\right)^{2}+\left(\frac{\partial}{\partial y}\right)^{2}\right)
$$

acting over the Hilbert space composed of all $\Gamma$-automorphic functions which are square integrable with respect to the hyperbolic measure, where

$$
\Gamma \cong \mathrm{SL}(2, \mathbb{Z}) /\{+1,-1\} \text {. }
$$

Let $\left\{\psi_{j}\right\}$ be a maximal orthonormal system such that $\Delta \psi_{j}=\lambda_{j} \psi_{j}$ for each $j \geq 1$ and $T(n) \psi_{j}=t_{j}(n) \psi_{j}$ for each integer $n \in \mathbb{N}$, where

$$
(T(n) f)(z)=\frac{1}{\sqrt{n}} \sum_{a d=n} \sum_{b=1}^{d} f\left(\frac{a z+b}{d}\right)
$$

is the Hecke operator. We shall further assume that $\psi_{j}(-\bar{z})=\varepsilon_{j} \psi_{j}(z)$ with $\varepsilon_{j}= \pm 1$. We then define $(s=\sigma+i t$ will denote a complex variable)

$$
H_{j}(s)=\sum_{n=1}^{\infty} t_{j}(n) n^{-s} \quad(\sigma>1),
$$

which we call the Hecke series associated with the Maass wave form $\psi_{j}(z)$,

2000 Mathematics Subject Classification: Primary 11M06; Secondary 11F72. 
and which can be continued to an entire function. As usual we put

$$
\alpha_{j}=\left|\varrho_{j}(1)\right|^{2}\left(\cosh \pi \kappa_{j}\right)^{-1},
$$

where $\varrho_{j}(1)$ is the first Fourier coefficient of $\psi_{j}(z)$. We note (see [15]) that

$$
\sum_{\kappa_{j} \leq K} \alpha_{j} H_{j}^{3}(1 / 2) \ll K^{2} \log ^{C} K \quad(C>0),
$$

that $H_{j}(1 / 2) \geq 0$ (see Katok-Sarnak [12]), and that there are $\asymp K$ eigenvalues $\kappa_{j}$ in $[K-1, K+1]$.

The function $\mathcal{Z}_{2}(s)$ was introduced by Y. Motohashi [14] (see also [8] and $[15])$, who showed that it has meromorphic continuation over $\mathbb{C}$. It is defined as

$$
\mathcal{Z}_{2}(s)=\int_{1}^{\infty}|\zeta(1 / 2+i x)|{ }^{4} x^{-s} d x \quad(\sigma>1) .
$$

He also established that in the half-plane $\sigma=\operatorname{Re} s>0$ it has the following singularities: the pole $s=1$ of order five, simple poles at $s=1 / 2 \pm i \kappa_{j}\left(\kappa_{j}=\right.$ $\left.\sqrt{\lambda_{j}-1 / 4}\right)$ and poles at $s=\varrho / 2$, where $\varrho$ denotes complex zeros of $\zeta(s)$. The residue of $\mathcal{Z}_{2}(s)$ at $s=1 / 2+i \kappa_{h}$ equals

$$
\begin{aligned}
R\left(\kappa_{h}\right)= & \sqrt{\frac{\pi}{2}}\left(2^{-i \kappa_{h}} \frac{\Gamma\left(1 / 4-i \kappa_{h} / 2\right)}{\Gamma\left(1 / 4+i \kappa_{h} / 2\right)}\right)^{3} \\
& \times \Gamma\left(2 i \kappa_{h}\right) \cosh \left(\pi \kappa_{h}\right) \sum_{\kappa_{j}=\kappa_{h}} \alpha_{j} H_{j}^{3}(1 / 2),
\end{aligned}
$$

and the residue at $s=1 / 2-i \kappa_{h}$ equals $\overline{R\left(\kappa_{h}\right)}$. Thus there is an intrinsic connection between $\mathcal{Z}_{2}(s)$ and $\alpha_{j} H_{j}^{3}(1 / 2)$, and therefore also between the fourth moment of $|\zeta(1 / 2+i x)|$ and $\alpha_{j} H_{j}^{3}(1 / 2)$. The plan of the paper is as follows: in Section 2 the modified Mellin transform is studied and five lemmas needed in what follows are proved. Mean square results on $\mathcal{Z}_{2}(s)$ are studied in Section 3, and two conjectures on $\mathcal{Z}_{2}(s)$ and their corollaries are given in Section 4. In Section 5 a conjecture on exponential sums with $\alpha_{j} H_{j}^{3}(1 / 2)$ is made. A new pointwise bound for $\mathcal{Z}_{2}(s)$ is obtained in Section 6 . Finally in Section 7 the general function $\mathcal{Z}_{k}(s)$ is studied.

2. The modified Mellin transform. The function $\mathcal{Z}_{2}(s)$ represents a sort of a modified Mellin transform of $|\zeta(1 / 2+i x)|^{4}$, where the Mellin transform of an integrable function $f(x)$ is commonly defined as

$$
\mathcal{M}[f(x)]=F(s)=\int_{0}^{\infty} x^{s-1} f(x) d x \quad(s=\sigma+i t) .
$$

Mellin transforms play an important rôle in Analytic Number Theory. By a change of variable they can be viewed as special cases of Fourier transforms, 
and their theory built by using the theory of Fourier transforms, for which the reader is referred to E. C. Titchmarsh's classical monograph [16]. Namely if $F(s)=\mathcal{M}[f(x)]$, then $\left(\xi=e^{x}\right)$

$$
F(\sigma+i t)=\int_{0}^{\infty} \xi^{\sigma+i t-1} f(\xi) d \xi=\int_{-\infty}^{\infty} e^{i x t} f\left(e^{x}\right) e^{\sigma x} d x
$$

is the Fourier transform of $f\left(e^{x}\right) e^{\sigma x}$.

An important feature of Mellin transforms is the so-called inversion formula. It states that if $F(s)=\mathcal{M}[f(x)], y^{\sigma-1} f(y) \in L^{1}(0, \infty)$ and $f(y)$ is of bounded variation in a neighbourhood of $y=x$, then

$$
\begin{aligned}
\frac{f(x+0)+f(x-0)}{2} & =\frac{1}{2 \pi i} \int_{(\sigma)} F(s) x^{-s} d s \\
& =\frac{1}{2 \pi i} \lim _{T \rightarrow \infty} \int_{\sigma-i T}^{\sigma+i T} F(s) x^{-s} d s .
\end{aligned}
$$

We recall that if $f(x)$ denotes measurable functions, then

$$
L^{p}(a, b):=\left\{\left.f(x)\left|\int_{a}^{b}\right| f(x)\right|^{p} d x<\infty\right\} .
$$

The Mellin inversion formula is usually derived from the inversion formula for Fourier transforms. Namely, if

$$
\widehat{g}(y)=\int_{-\infty}^{\infty} e^{i x y} g(x) d x
$$

is the Fourier transform of $g(x)$, then under suitable conditions this is equivalent to

$$
g(x)=\frac{1}{2 \pi} \int_{-\infty}^{\infty} e^{-i x y} \widehat{g}(y) d y .
$$

For example if $g, \widehat{g} \in L^{1}(-\infty, \infty)$, then (2.2) holds for almost all $x \in$ $(-\infty, \infty)$. If additionally $g$ is continuous in $(-\infty, \infty)$, then $(2.2)$ holds for all $x \in(-\infty, \infty)$. A variant of Parseval's formula for Fourier transforms is the identity

$$
\frac{1}{2 \pi} \int_{-\infty}^{\infty}|\widehat{f}(x)|^{2} d x=\int_{-\infty}^{\infty}|f(x)|^{2} d x
$$

and it can be used to derive Parseval's formula for Mellin transforms. 
The modified Mellin transform $m[f(x)]$, of which $\mathcal{Z}_{2}(s)$ is a special case, will be now defined as

$$
F^{*}(s)=m[f(x)]=\int_{1}^{\infty} f(x) x^{-s} d x \quad(s=\sigma+i t),
$$

which is often more convenient to have in applications than the ordinary Mellin transform, since now there are no convergence problems at $x=0$. If $\bar{f}(x)=f(1 / x)$ when $0<x \leq 1$ and $\bar{f}(x)=0$ otherwise, then

$$
m[f(x)]=\mathcal{M}\left[\frac{1}{x} \bar{f}(x)\right]
$$

so that the properties of $m[f(x)]$ can be deduced from the properties of the ordinary Mellin transform $\mathcal{M}[f(x)]$ by the use of (2.5). In what follows five lemmas concerning the properties of the modified Mellin transform will be proved.

Lemma 1. If $x^{-\sigma} f(x) \in L^{1}(1, \infty)$ and $f(x)$ is continuous for $x>1$, then

$$
f(x)=\frac{1}{2 \pi i} \int_{(\sigma)} F^{*}(s) x^{s-1} d s, \quad F^{*}(s)=m[f(x)] .
$$

Proof. If $F^{*}(s)=m[f(x)]$, then from (2.5) and (2.1) we have

$$
\frac{1}{x} \bar{f}(x)=\frac{1}{2 \pi i} \int_{(\sigma)} F^{*}(s) x^{-s} d s
$$

provided that $y^{\sigma-1} \frac{1}{y} \bar{f}(y) \in L^{1}(0, \infty)$. This means that

$$
\int_{0}^{1} y^{\sigma-1} \frac{1}{y}\left|f\left(\frac{1}{y}\right)\right| d y<\infty,
$$

and making the change of variable $y=1 / x$, we deduce that the last condition is equivalent to $x^{-\sigma} f(x) \in L^{1}(1, \infty)$. Changing $x$ to $1 / x$ in $(2.7)$ we then obtain (2.6).

Lemma 2. If $F^{*}(s)=m[f(x)], G^{*}(s)=m[g(x)]$, and $f(x), g(x)$ are real-valued, continuous functions for $x>1$, such that

$$
x^{1 / 2-c} f(x) \in L^{2}(1, \infty), \quad x^{c-1 / 2-\sigma} g(x) \in L^{2}(1, \infty),
$$

then

$$
m[f(x) g(x)]=\frac{1}{2 \pi i} \int_{(c)} F^{*}(w) G^{*}(s+1-w) d w .
$$


Proof. From [16, Theorem 73] we have

$$
\int_{0}^{\infty} f(x) g(x) x^{s-1} d x=\frac{1}{2 \pi i} \int_{(c)} F(w) G(s-w) d w
$$

if

$$
\begin{gathered}
F(s)=\mathcal{M}[f(x)], \quad G(s)=\mathcal{M}[g(x)], \\
x^{c-1 / 2} f(x) \in L^{2}(0, \infty), \quad x^{\sigma-c-1 / 2} g(x) \in L^{2}(0, \infty) .
\end{gathered}
$$

In place of $f(x)$ and $g(x)$ in (2.9) we shall take $\frac{1}{x} \bar{f}(x)$ and $\bar{g}(x)$, respectively. By (2.5) we have $\mathcal{M}\left[\frac{1}{x} \bar{f}(x)\right]=F^{*}(s)$ and

$$
\mathcal{M}[\bar{g}(x)]=\int_{0}^{1} g\left(\frac{1}{x}\right) x^{s-1} d x=G^{*}(s+1) .
$$

Consequently, (2.9) gives

$$
\int_{0}^{\infty} \frac{1}{x} \bar{f}(x) \bar{g}(x) x^{s-1} d x=\frac{1}{2 \pi i} \int_{(c)} F^{*}(w) G^{*}(s+1-w) d w .
$$

After the change of variable $y=1 / x$ this reduces to $(2.8)$ if

$$
x^{c-1 / 2} \cdot \frac{1}{x} \bar{f}(x) \in L^{2}(0, \infty), \quad x^{\sigma-c-1 / 2} \cdot \frac{1}{x} \bar{g}(x) \in L^{2}(0, \infty),
$$

and these conditions are easily seen to be equivalent to

$$
x^{1 / 2-c} f(x) \in L^{2}(1, \infty), \quad x^{c-1 / 2-\sigma} g(x) \in L^{2}(1, \infty) .
$$

Lemma 3. If $F^{*}(s)=m[f(x)], G^{*}(s)=m[g(x)]$, and $f(x), g(x)$ are real-valued, continuous functions for $x>1$, such that

$$
x^{1 / 2-\sigma} f(x) \in L^{2}(1, \infty), \quad x^{1 / 2-\sigma} g(x) \in L^{2}(1, \infty),
$$

then

$$
\int_{1}^{\infty} f(x) g(x) x^{1-2 \sigma} d x=\frac{1}{2 \pi i} \int_{(\sigma)} F^{*}(s) \overline{G^{*}(s)} d s .
$$

Proof. This follows similarly to the preceding proof from Parseval's formula for Mellin transforms in the form

if

$$
\int_{0}^{\infty} f(x) g(x) x^{2 \sigma-1} d x=\frac{1}{2 \pi i} \int_{(\sigma)} F(s) \overline{G(s)} d s
$$

$$
\begin{aligned}
F(s) & =\mathcal{M}[f(x)], & & G(s)=\mathcal{M}[g(x)], \\
x^{\sigma-1 / 2} f(x) & \in L^{2}(0, \infty), & & x^{\sigma-1 / 2} g(x) \in L^{2}(0, \infty) .
\end{aligned}
$$

The last relation follows e.g. from [16, Theorem 72] by a change of variable. 
Lemma 3, in the special case $f(x)=g(x)$, is a natural tool for investigating mean square formulas connected with the modified Mellin transform. In particular, it offers the possibility to obtain mean square estimates of $f(x)$ from the mean square estimates of $m[f(x)]$, provided we have adequate analytic information about the latter. A result in this direction, which is useful for the applications that we have mind, is now given as

Lemma 4. Suppose that $g(x)$ is a real-valued, integrable function on $[a, b]$, a subinterval of $[2, \infty)$, which is not necessarily finite. Then

$$
\int_{0}^{T}\left|\int_{a}^{b} g(x) x^{-s} d x\right|^{2} d t \leq 2 \pi \int_{a}^{b} g^{2}(x) x^{1-2 \sigma} d x \quad(s=\sigma+i t, T>0, a<b) .
$$

Proof. Let

$$
I:=\int_{0}^{T}\left|\int_{a}^{b} g(x) x^{-s} d x\right|^{2} d t \quad(s=\sigma+i t, T>0) .
$$

In Lemma 3 set $f(x)=g(x)$ if $a \leq x \leq b$ and $f(x)=0$ otherwise. Then

$$
F^{*}(s)=m[f(x)]=\int_{a}^{b} g(x) x^{-s} d x, \quad F^{*}(s)=G^{*}(s),
$$

and $x^{1 / 2-\sigma} f(x) \in L^{2}(1, \infty)$ for any $\sigma$. Consequently, (2.10) of Lemma 3 (with $f \equiv g$ ) gives

$$
\frac{I}{2 \pi} \leq \frac{1}{2 \pi} \int_{-\infty}^{\infty}\left|F^{*}(\sigma+i t)\right|^{2} d t=\int_{a}^{b} g^{2}(x) x^{1-2 \sigma} d x
$$

and (2.11) follows from (2.12) and (2.13).

In case we have sufficient information on $f$ and $F^{*}$, we can obtain an explicit bound for the mean square estimate of $f(x)$ :

Lemma 5. Let $f(x) \in C^{\infty}[2, \infty)$ be a real-valued function for which

(a) $\int_{1}^{X}\left|f^{(r)}(x)\right| d x \ll_{\varepsilon, r} X^{1+\varepsilon}(r=0,1,2, \ldots)$ and

(b) $F^{*}(s)=m[f(x)]$ has a pole at $s=1$ of order $l$, but otherwise can be analytically continued to the region $\operatorname{Re} s>1 / 2$, where it is of polynomial growth in $|\operatorname{Im} s|$.

Then for $1 / 2<\sigma<1$ and any given $\varepsilon>0$ we have

$$
\begin{aligned}
& \int_{T}^{2 T} f^{2}(x) d x \\
& \quad \ll_{\varepsilon} \log ^{l-1} T \cdot \int_{T / 2}^{5 T / 2}|f(x)| d x+T^{2 \sigma-1} \int_{0}^{T^{1+\varepsilon}}\left|F^{*}(\sigma+i t)\right|^{2} d t .
\end{aligned}
$$


Proof. Let $\varphi(x) \in C^{\infty}(0, \infty)$ be a test function such that $\varphi(x) \geq 0$, $\varphi(x)=1$ for $T \leq x \leq 2 T, \varphi(x)=0$ for $x<T / 2$ or $x>5 T / 2\left(T \geq T_{0}>0\right)$, $\varphi(x)$ is increasing in $[T / 2, T]$ and decreasing in $[2 T, 5 T / 2]$. Then obviously

$$
I_{1} \leq I_{2}
$$

where

$$
I_{1}:=\int_{T}^{2 T} f^{2}(x) d x, \quad I_{2}:=\int_{T / 2}^{5 T / 2} \varphi(x) f^{2}(x) d x .
$$

From the assumption (a) we have $x^{-\sigma} f(x) \in L^{1}(1, \infty)$ if $\sigma>1$. Therefore Lemma 1 gives

$$
\begin{aligned}
f(x) & =\frac{1}{2 \pi i} \int_{(1+\varepsilon)} F^{*}(w) x^{w-1} d w \\
& =\frac{1}{2 \pi i} \int_{\mathcal{L}} F^{*}(w) x^{w-1} d w+Q_{l-1}(\log x),
\end{aligned}
$$

where $Q_{l-1}(\log x)$ is a polynomial in $x$ of degree $l-1$, and $\mathcal{L}$ is the line Re $w=1+\varepsilon$ with a small indentation to the left at the pole $w=1$ of $F^{*}(w)$ of order $l$, so that by the residue theorem we pick a contribution equal to $Q_{l-1}(\log x)$. Therefore $(2.15)$ yields

$$
\begin{aligned}
I_{2}= & \frac{1}{2 \pi i} \int_{\mathcal{L}} F^{*}(w)\left(\int_{T / 2}^{5 T / 2} \varphi(x) f(x) x^{w-1} d x\right) d w \\
& +O\left(\log ^{l-1} T \cdot \int_{T / 2}^{5 T / 2} \varphi(x)|f(x)| d x\right) .
\end{aligned}
$$

We integrate $r$ times by parts to obtain

$$
\begin{aligned}
\int_{T / 2}^{5 T / 2} \varphi(x) f(x) x^{w-1} d x & \\
& =\frac{(-1)^{r}}{w \ldots(w+r-1)} \int_{T / 2}^{5 T / 2}(\varphi(x) f(x))^{(r)} x^{w+r-1} d x .
\end{aligned}
$$

Since $\varphi^{(r)}(x) \ll_{r} T^{-r}$ and (a) of the lemma holds, by (2.17) it follows that the portion of the integral over $w$ in (2.16) for which $|v|>T^{1+\varepsilon}$ makes a negligible contribution, namely $\ll T^{-A}$ for any given $A>0$, provided that we choose $r=r(A, \varepsilon)$ sufficiently large. This remains true even if we move the contour of integration to the left. Hence if $0<u<1 / 2, w=u+i v$, then 
by (2.16) and the Cauchy-Schwarz inequality for integrals we infer that

$$
\begin{aligned}
I_{2}= & \frac{1}{2 \pi} \int_{-T^{1+\varepsilon}}^{T^{1+\varepsilon}} F^{*}(u+i v) \int_{T / 2}^{5 T / 2} \varphi(x) f(x) x^{u+i v-1} d x d v \\
& +O\left(\log ^{l-1} T \cdot \int_{T / 2}^{5 T / 2} \varphi(x)|f(x)| d x\right) \\
\ll & \left\{\int_{0}^{T^{1+\varepsilon}}\left|F^{*}(u+i v)\right|^{2} d v \int_{0}^{T^{1+\varepsilon}}\left|\int_{T / 2}^{5 T / 2} \varphi(x) f(x) x^{u+i v-1} d x\right|^{2} d v\right\}^{1 / 2} \\
& +\log ^{l-1} T \cdot \int_{T / 2}^{5 T / 2} \varphi(x)|f(x)| d x .
\end{aligned}
$$

Now we apply Lemma 4 (with $g(x)=\varphi(x) f(x), s=1-u+i v, T$ replaced by $\left.T^{1+\varepsilon}\right)$ to deduce that

$$
\begin{aligned}
\int_{0}^{T^{1+\varepsilon}} \mid & \left.\int_{T / 2}^{5 T / 2} \varphi(x) f(x) x^{u+i v-1} d x\right|^{2} d v \\
& \ll \int_{T / 2}^{5 T / 2} \varphi^{2}(x) f^{2}(x) x^{2 u-1} d x \ll T^{2 u-1} \int_{T / 2}^{5 T / 2} \varphi(x) f^{2}(x) d x=T^{2 u-1} I_{2},
\end{aligned}
$$

since $0 \leq \varphi(x) \leq 1$. It follows that

$$
I_{2} \ll \log ^{l-1} T \cdot \int_{T / 2}^{5 T / 2} \varphi(x)|f(x)| d x+\left(\int_{0}^{T^{1+\varepsilon}}\left|F^{*}(u+i v)\right|^{2} d v \cdot T^{2 u-1} I_{2}\right)^{1 / 2},
$$

which easily gives (2.11) with $\sigma=u$.

3. The mean square of $\mathcal{Z}_{2}(s)$. Mean square problems involving $\mathcal{Z}_{2}(s)$ are naturally of interest. They were investigated in [8], where it was shown that

$$
\int_{0}^{T}\left|\mathcal{Z}_{2}(\sigma+i t)\right|^{2} d t \ll T^{(10-8 \sigma) / 3} \log ^{C} T \quad(1 / 2<\sigma<1, C>0) .
$$

We now introduce the function $E_{2}(T)$, the error term in the asymptotic formula for the mean fourth power of $\zeta(1 / 2+i t)$, customarily defined by the relation

$$
\int_{0}^{T}|\zeta(1 / 2+i t)|^{4} d t=T P_{4}(\log T)+E_{2}(T)
$$


with

$$
P_{4}(x)=\sum_{j=0}^{4} a_{j} x^{j}, \quad a_{4}=\frac{1}{2 \pi^{2}} .
$$

For some recent results on $E_{2}(T)$ see [3]-[6], [9], [10], [14] and [15]. For the explicit evaluation of the $a_{j}$ 's in (3.3), see [4]. A fundamental result in the theory of $E_{2}(T)$ is the mean square estimate

$$
\int_{0}^{T} E_{2}^{2}(t) d t \ll_{\varepsilon} T^{2+\varepsilon},
$$

where $\varepsilon$ denotes arbitrarily small positive constants, not necessarily the same at each occurrence. In fact, Ivić-Motohashi [9] proved (3.4) with $\log ^{C} T$ in place of $T^{\varepsilon}$, but for our present purpose (3.4) suffices; the integral in (3.4) is actually $\gg T^{2}$, as shown in [6], so that the bound in (3.4) is essentially best possible. We have (see [5], [6], [10] and [15])

$$
E_{2}(T) \ll T^{2 / 3} \log ^{8} T, \quad E_{2}(T)=\Omega_{ \pm}(\sqrt{T})
$$

and

$$
\int_{0}^{T} E_{2}(t) d t=O\left(T^{3 / 2}\right), \quad \int_{0}^{T} E_{2}(t) d t=\Omega_{ \pm}\left(T^{3 / 2}\right) .
$$

Thus if $c$ is a constant for which

$$
E_{2}(T) \ll_{\varepsilon} T^{c+\varepsilon},
$$

then (3.5) implies that one must have $1 / 2 \leq c \leq 2 / 3$. It was proved in [8] that, besides (3.1), one also has

$$
\int_{0}^{T}\left|\mathcal{Z}_{2}(\sigma+i t)\right|^{2} d t \ll_{\varepsilon} T^{\varepsilon}\left(T+T^{(2-2 \sigma) /(1-c)}\right) \quad(1 / 2<\sigma<1) .
$$

We can derive a mean square result on $\mathcal{Z}_{2}(s)$ by the use of Lemma 3 . Namely from (3.2) we obtain, by integration by parts, the representation

$$
\begin{aligned}
\mathcal{Z}_{2}(s) & =\int_{1}^{\infty}\left(P_{4}(\log x)+P_{4}^{\prime}(\log x)+E_{2}^{\prime}(x)\right) x^{-s} d x \\
& =\sum_{j=0}^{5} c_{j}(s-1)^{-j}+s \int_{1}^{\infty} E_{2}(x) x^{-s-1} d x .
\end{aligned}
$$

It follows from (3.4) and the Cauchy-Schwarz inequality for integrals that the function

$$
z_{2}(s):=\mathcal{Z}_{2}(s)-\sum_{j=0}^{5} c_{j}(s-1)^{-j}=s \int_{1}^{\infty} E_{2}(x) x^{-s-1} d x
$$


is regular for $\sigma>1 / 2$, where the constants $c_{j}$ may be explicitly evaluated in terms of the $a_{j}$ 's in (3.3). Then Lemma 3 gives

$$
\int_{1}^{\infty} E_{2}^{2}(x) x^{-1-2 \sigma} d x=\frac{1}{2 \pi} \int_{-\infty}^{\infty}\left|\frac{z_{2}(\sigma+i t)}{\sigma+i t}\right|^{2} d t \quad(\sigma>1 / 2) .
$$

Thus, for $1 / 2<\sigma<1$, we have

$$
1 \gg \int_{T}^{2 T}\left|\frac{z_{2}(\sigma+i t)}{\sigma+i t}\right|^{2} d t \gg T^{-2} \int_{T}^{2 T}\left|z_{2}(\sigma+i t)\right|^{2} d t
$$

which yields

$$
\begin{array}{rl}
\int_{T}^{2 T}\left|\mathcal{Z}_{2}(\sigma+i t)\right|^{2} & d t \\
& \ll \int_{T}^{2 T}\left(\left|z_{2}(\sigma+i t)\right|^{2}+1\right) d t \ll T^{2} \quad(1 / 2<\sigma<1) .
\end{array}
$$

Note that if (3.12) is known, then (3.11) yields (3.4). To see this let $s=\sigma+i t$ with $1 / 2<\sigma<1, t \geq t_{0}>0$. Then by the residue theorem

$$
\mathcal{Z}_{2}(s)=\frac{1}{2 \pi i} \int_{\mathcal{D}} X^{w} \Gamma(w) \mathcal{Z}_{2}(w+s) d w \quad\left(2 \leq X \ll t^{A}\right),
$$

where $\mathcal{D}$ is the rectangle with vertices $1 / 2-\sigma+\varepsilon \pm i \log ^{2} t, 1-\sigma+\varepsilon \pm i \log ^{2} t$ and $0<\varepsilon<\sigma-1 / 2$. By Stirling's formula for the gamma-function it follows then that

(3.14) $\mathcal{Z}_{2}(s) \ll_{\varepsilon} X^{1 / 2-\sigma+\varepsilon} \int_{-\log ^{2} t}^{\log ^{2} t} e^{-|v|}\left|\mathcal{Z}_{2}(1 / 2+\varepsilon+i v+i t)\right| d v+X^{1-\sigma+\varepsilon}$,

since $\mathcal{Z}_{2}(s) \ll_{\sigma} 1$ for $\sigma>1$. If we additionally suppose that $T \leq t \leq 2 T$, then from (3.12) (with $\sigma=1 / 2+\varepsilon$ ) and (3.14) we obtain

$$
\begin{aligned}
& \int_{T}^{2 T}\left|\mathcal{Z}_{2}(\sigma+i t)\right|^{2} d t \\
& \quad \ll_{\varepsilon} T X^{2-2 \sigma+\varepsilon}+X^{1-2 \sigma+\varepsilon} \int_{-\log ^{2} T}^{\log ^{2} T} e^{-|v|} \int_{T}^{2 T}\left|\mathcal{Z}_{2}(1 / 2+\varepsilon+i v+i t)\right|^{2} d t d v \\
& \ll_{\varepsilon} T X^{2-2 \sigma+\varepsilon}+T^{2} X^{1-2 \sigma+\varepsilon} \ll_{\varepsilon} T^{3-2 \sigma+\varepsilon}
\end{aligned}
$$

if we choose $X=T$. Hence

$$
\int_{1}^{T}\left|\mathcal{Z}_{2}(\sigma+i t)\right|^{2} d t \ll_{\varepsilon} T^{3-2 \sigma+\varepsilon} \quad(1 / 2<\sigma<1) .
$$


Since $3-2 \sigma<2$ for $\sigma>1 / 2,(3.15)$ yields

$$
\int_{-\infty}^{\infty}\left|\frac{z_{2}(\sigma+i t)}{\sigma+i t}\right|^{2} d t \ll 1 \quad(1 / 2<\sigma<1)
$$

and consequently (3.11) yields (3.4) with $\sigma=(1+\varepsilon) / 2$. As proved in [9], (3.4) then easily gives

$$
E_{2}(T) \ll_{\varepsilon} T^{2 / 3+\varepsilon}, \quad \int_{0}^{T}|\zeta(1 / 2+i t)|^{12} d t \ll_{\varepsilon} T^{2+\varepsilon},
$$

and both of these estimates are best known up to " $\varepsilon$ ". This shows the strength and importance of mean square estimates for $\mathcal{Z}_{2}(s)$ (see also (3.29)).

A natural problem is to ask: what is the true order of magnitude of

$$
I_{\sigma}(T):=\int_{1}^{T}\left|\mathcal{Z}_{2}(\sigma+i t)\right|^{2} d t \quad(\sigma>1 / 2) ?
$$

It seems very hard to speculate what the shape of the asymptotic formula for $I_{\sigma}(T)$ ought to be for fixed $\sigma>1 / 2$, not to mention values like $\sigma=$ $1 / 2+1 / \log T$ (in view of the poles $s=1 / 2 \pm i \kappa_{j}$ of $\mathcal{Z}_{2}(s)$ ) etc. The lower limit of integration in (3.17) is unity and not zero for technical reasons, in view of the pole $s=1$ of $\mathcal{Z}_{2}(s)$. The bound

$$
I_{1 / 2+\varepsilon}(T) \ll T^{2}
$$

follows from (3.15) and in view of the fact that (3.4) is essentially best possible, it follows that by using Parseval's formula, namely the identity (3.11), we cannot obtain a stronger estimate than (3.18). Nevertheless there seems to be no apparent reason why (3.18) could not be improved. It can also be remarked that (3.18), via the second bound in (3.16) and the CauchySchwarz inequality for integrals, yields the bound

$$
\int_{0}^{T}|\zeta(1 / 2+i t)|^{8} d t \ll_{\varepsilon} T^{3 / 2+\varepsilon},
$$

which is best known up to " $\varepsilon$ ". Thus any improvement of (3.18) would have important consequences in zeta-function theory. On the other hand it is of interest to obtain lower bounds (i.e., omega-results) for $I_{\sigma}(T)$. In this direction we have

TheOREM 1. For any given $\varepsilon>0$ and fixed $\sigma$ satisfying $1 / 2<\sigma<1$ we have

$$
\int_{1}^{T}\left|\mathcal{Z}_{2}(\sigma+i t)\right|^{2} d t \gg_{\varepsilon} T^{2-2 \sigma-\varepsilon} .
$$


Proof. Let, as usual,

$$
\begin{gathered}
Z(t):=\chi^{-1 / 2}(1 / 2+i t) \zeta(1 / 2+i t), \\
\chi(s)=\frac{\zeta(s)}{\zeta(1-s)}=\frac{\pi^{s-1 / 2} \Gamma(1 / 2-s / 2)}{\Gamma(s / 2)} .
\end{gathered}
$$

Then $Z(t) \in \mathbb{R}$ if $t \in \mathbb{R},|Z(t)|=|\zeta(1 / 2+i t)|$, and $Z^{4}(t)$ and all its derivatives are bounded in mean by a suitable log-power. This follows by Hölder's inequality for integrals, and the fact that (see [11, Chapter 3])

$$
\begin{aligned}
Z^{(k)}(t)= & O_{k}\left(t^{-1 / 4}\left(\frac{3}{2} \log t\right)^{k+1}\right) \\
& +2 \sum_{n \leq \sqrt{t /(2 \pi)}} n^{-1 / 2}\left(\log \frac{\sqrt{t /(2 \pi)}}{n}\right)^{k} \\
& \times \cos \left(t \log \frac{\sqrt{t /(2 \pi)}}{n}-\frac{t}{2}-\frac{\pi}{8}+\frac{\pi k}{2}\right) .
\end{aligned}
$$

Another way to see this is to use Leibniz's formula for the derivative of a product, the expression for $\chi(s)$ and properties of the gamma-function. If we set $f(x)=Z^{4}(x)$, then $F^{*}(s)=\mathcal{Z}_{2}(s)$, and the assumptions (a) and (b) of Lemma 5 are satisfied $\left(l=5, \mathcal{Z}_{2}(s)\right.$ is of polynomial growth for $\sigma>1 / 2$ by (3.9)). Therefore (2.14) gives, for $1 / 2<\sigma<1$,

$$
\begin{aligned}
& \int_{T}^{2 T}|\zeta(1 / 2+i t)|^{8} d t \ll_{\varepsilon} \log ^{4} T \cdot \int_{T / 2}^{5 T / 2}|\zeta(1 / 2+i t)|^{4} d t \\
& +T^{2 \sigma-1} \int_{0}^{T^{1+\varepsilon}}\left|\mathcal{Z}_{2}(\sigma+i t)\right|^{2} d t .
\end{aligned}
$$

But (see [3, Theorem 6.5]) we have

$$
\int_{T}^{2 T}|\zeta(1 / 2+i t)|^{8} d t \gg T \log ^{16} T,
$$

hence (3.21) yields

$$
T \log ^{16} T \ll_{\varepsilon} T^{2 \sigma-1} \int_{0}^{T^{1+\varepsilon}}\left|\mathcal{Z}_{2}(\sigma+i t)\right|^{2} d t .
$$

The lower bound in (3.20) then follows if we substitute $T$ for $T^{1+\varepsilon}$ in (3.22).

The mean square of $\mathcal{Z}_{2}(s)$ has a convexity property, embodied in 
Theorem 2. For fixed $c, \sigma$ such that $1 / 2<c \leq \sigma$ and $t \geq T \geq 3$ we have

$$
\begin{aligned}
& \int_{T}^{2 T}\left|\mathcal{Z}_{2}(\sigma+i t)\right|^{2} d t \\
& \ll_{\varepsilon} T^{-1}+T^{\varepsilon}\left(T^{2 c-2 \sigma} \int_{T}^{2 T}\left|\mathcal{Z}_{2}(c+i t)\right|^{2} d t+T^{2-2 \sigma}+T^{5-6 \sigma}\right), \\
& \mathcal{Z}_{2}(\sigma+i t) \ll_{\varepsilon} t^{-1}+t^{c-\sigma+\varepsilon} \int_{t-t^{\varepsilon}}^{t+t^{\varepsilon}}\left|\mathcal{Z}_{2}(c+i v)\right| d v+t^{3-4 \sigma} \log ^{4} t .
\end{aligned}
$$

Proof. The proof of both bounds is similar (for (3.24) we take $X=t^{4}$ ), so only (3.23) will be considered in detail. Clearly we may assume that $c<\sigma$. Let $T \leq t \leq 2 T \leq X \leq T^{A}$, where $A(>1)$ is a constant. For $\sigma>1$ we have

$$
\begin{aligned}
\mathcal{Z}_{2}(s)= & \int_{1}^{2 T^{1-\varepsilon}} \varrho(x)|\zeta(1 / 2+i x)|^{4} x^{-s} d x+\int_{T^{1-\varepsilon}}^{2 X} \sigma(x)|\zeta(1 / 2+i x)|^{4} x^{-s} d x \\
& +\int_{X}^{\infty} \tau(x)|\zeta(1 / 2+i x)|^{4} x^{-s} d x \\
= & I_{1}(s)+I_{2}(s)+I_{3}(s),
\end{aligned}
$$

say. Here $\varrho(x), \sigma(x), \tau(x) \in C^{\infty}$ are nonnegative functions such that: $\varrho(x)=1$ for $1 \leq x \leq T^{1-\varepsilon}, \varrho(x)=0$ for $x \geq 2 T^{1-\varepsilon}, \sigma(x)=0$ for $x \geq 2 X$, $\tau(x)=1-\sigma(x)$. By repeated integration by parts, as in the proof of (3.21), we obtain

$$
I_{1}(s)=\frac{|\zeta(1 / 2+i)|^{4}}{s-1}+O\left(t^{-2}\right) \ll \frac{1}{T} .
$$

Next note that

$$
\begin{aligned}
I_{3}(s)= & \int_{X}^{\infty} \tau(x) Q_{4}(\log x) x^{-s} d x+\int_{X}^{\infty} \tau(x) E_{2}^{\prime}(x) x^{-s} d x \\
= & \frac{1}{s-1} \int_{X}^{\infty} x^{1-s}\left(\tau^{\prime}(x) Q_{4}(\log x)+\tau(x) x^{-1} Q_{4}^{\prime}(\log x)\right) d x \\
& -\int_{X}^{\infty} E_{2}(x)\left(\tau^{\prime}(x) x^{-s}-s \tau(x) x^{-s-1}\right) d x \\
= & I_{4}(s)-I_{5}(s),
\end{aligned}
$$

say. Since $\tau^{\prime}(x) \ll X^{-1}$ and $\tau^{\prime}(x)=0$ for $x \geq 2 X$, it follows that 


$$
\begin{aligned}
I_{4}(s)= & \frac{1}{s-1} \int_{X}^{2 X} x^{1-s}\left(\tau^{\prime}(x) Q_{4}(\log x)+\tau(x) x^{-1} Q_{4}^{\prime}(\log x)\right) d x \\
& +\frac{1}{s-1} \int_{2 X}^{\infty} x^{-s} Q_{4}^{\prime}(\log x) d x \\
= & I_{6}(s)+I_{7}(s),
\end{aligned}
$$

say. The function $I_{6}(s)$ is regular for $s \neq 1$ and for $T \leq t \leq 2 T$ it is $\ll T^{-1} X^{1-\sigma} \log ^{4} X$. We can evaluate $I_{7}(s)$ and obtain

$$
I_{7}(s)=\frac{c(2 X)^{1-s}}{(s-1)^{4}}
$$

which provides analytic continuation of $I_{7}(s)$ to $\mathbb{C}$. In view of $(3.4)$ (or $(3.6)$ ) $I_{5}(s)$ is regular for $\sigma>1 / 2$, and therefore we obtain

$$
\int_{T}^{2 T}\left|I_{5}(s)\right|^{2} d t \ll T^{2} \int_{X}^{\infty} E_{2}^{2}(x) x^{-1-2 \sigma} d x \ll T^{2} X^{1-2 \sigma} \log ^{C} X
$$

by using Lemma 4. Consequently, we have from (3.24) and (3.25), for $\sigma>1 / 2$,

$$
\begin{aligned}
\int_{T}^{2 T}\left(\left|I_{1}(s)\right|^{2}+\left|I_{3}(s)\right|^{2}\right) d t & \ll T^{-1}+T^{-1} X^{2-2 \sigma} \log ^{8} X+T^{2} X^{1-2 \sigma} \log ^{C} X \\
& \ll T^{-1}+T^{5-6 \sigma} \log ^{C} T
\end{aligned}
$$

for $X=T^{3}$, which we henceforth assume. It remains to deal with the mean square of $I_{2}(s)$, which we write as a sum of $O(\log T)$ integrals of the type

$$
F_{K}(s):=\int_{K / 2}^{5 K^{\prime} / 2} \varphi(x)|\zeta(1 / 2+i x)|^{4} x^{-s} d x \quad\left(T^{1-\varepsilon} \leq K<K^{\prime} \leq 2 K \ll X\right),
$$

where $\varphi(x) \in C^{\infty}$ is a nonnegative function supported in $\left[K / 2,5 K^{\prime} / 2\right]$ such that $\varphi(x)=1$ for $K<K^{\prime} \leq 2 K$, and

$$
\varphi^{(r)}(x) \ll_{r} K^{-r} \quad(r=0,1,2, \ldots) .
$$

To connect $F_{K}(s)$ and $\mathcal{Z}_{2}(s)$ note that from the Mellin inversion formula (2.6) we have

$$
|\zeta(1 / 2+i x)|^{4}=\frac{1}{2 \pi i} \int_{(1+\varepsilon)} \mathcal{Z}_{2}(s) x^{s-1} d s \quad(x>1) .
$$

Here we replace the line of integration by the contour $\mathcal{L}$, consisting of the same straight line from which the segment $[1+\varepsilon-i, 1+\varepsilon+i]$ is removed and replaced by a circular arc of unit radius, lying to the left of the line, which passes over the pole $s=1$ of the integrand. By the residue theorem we have 


$$
|\zeta(1 / 2+i x)|^{4}=\frac{1}{2 \pi i} \int_{\mathcal{L}} \mathcal{Z}_{2}(s) x^{s-1} d s+Q_{4}(\log x) \quad(x>1),
$$

where we have set $(\mathrm{cf} .(3.2)) Q_{4}(\log x)=P_{4}(\log x)+P_{4}^{\prime}(\log x)$. Hence by using (3.27) we obtain

$$
\begin{aligned}
F_{K}(s)= & \frac{1}{2 \pi i} \int_{\mathcal{L}} \mathcal{Z}_{2}(w)\left(\int_{K / 2}^{5 K^{\prime} / 2} \varphi(x) x^{w-s-1} d x\right) d w \\
& +\int_{K / 2}^{5 K^{\prime} / 2} \varphi(x) Q_{4}(\log x) x^{-s} d x .
\end{aligned}
$$

In view of (3.26) we infer, by repeated integration by parts, that the last integral in (3.28) is $\ll T^{-A}$ for any given $A>0$. Similarly we note that

$$
\begin{aligned}
& \int_{K / 2}^{5 K^{\prime} / 2} \varphi(x) x^{w-s-1} d x \\
& \quad=(-1)^{r} \int_{K / 2}^{5 K^{\prime} / 2} \varphi^{(r)}(x) \frac{x^{w-s+r-1}}{(w-s) \ldots(w-s+r-1)} d x \ll T^{-A}
\end{aligned}
$$

for any given $A>0$, provided that $|\operatorname{Im} w-\operatorname{Im} s|>T^{\varepsilon}$ and $r=r(A, \varepsilon)$ is sufficiently large. Thus if in the $w$-integral in (3.28) we replace the contour $\mathcal{L}$ by the straight line $\operatorname{Re} w=c$, we obtain

$$
F_{K}(s) \ll K^{c-\sigma} \int_{t-T^{\varepsilon}}^{t+T^{\varepsilon}}\left|\mathcal{Z}_{2}(c+i v)\right| d v+T^{-2},
$$

which gives

$$
\begin{aligned}
& \int_{T}^{2 T}\left|I_{2}(s)\right|^{2} d t \\
& \ll T^{-1}+\log T \max _{T^{1-\varepsilon} \ll K \ll T^{3}} K^{2 c-2 \sigma} \int_{T}^{2 T}\left(\int_{t-T^{\varepsilon}}^{t+T^{\varepsilon}}\left|\mathcal{Z}_{2}(c+i v)\right| d v\right)^{2} d t \\
& \ll T^{-1}+\log T \max _{T^{1-\varepsilon} \ll K \ll T^{3}} K^{2 c-2 \sigma} T^{\varepsilon} \int_{T}^{2 T} \int_{t-T^{\varepsilon}}^{t+T^{\varepsilon}}\left|\mathcal{Z}_{2}(c+i v)\right|^{2} d v d t \\
& \ll T^{-1}+\log T \max _{T^{1-\varepsilon} \ll K \ll T^{3}} K^{2 c-2 \sigma} T^{\varepsilon} \int_{T-T^{\varepsilon}}^{2 T+T^{\varepsilon}}\left|\mathcal{Z}_{2}(c+i v)\right|^{2} \int_{v-T^{\varepsilon}}^{v+T^{\varepsilon}} d t d v \\
& \ll T^{-1}+T^{2 c-2 \sigma+4 \varepsilon} \int_{T}^{2 T}\left|\mathcal{Z}_{2}(c+i v)\right|^{2} d v+T^{2-2 \sigma+4 \varepsilon},
\end{aligned}
$$


where we used the bound $\mathcal{Z}_{2}(\sigma+i t) \ll_{\varepsilon} t^{1-\sigma+\varepsilon}$ (see Theorem 3), whose proof is independent of the present theorem. Collecting the above bounds we obtain (3.23).

Corollary 1. For any given $\varepsilon>0$ we have

$$
\int_{T}^{2 T}\left|\mathcal{Z}_{2}(\sigma+i t)\right|^{2} d t \ll_{\varepsilon} T^{-1}+T^{8 / 3-2 \sigma+\varepsilon} \quad(\sigma \geq 5 / 6) .
$$

Proof. From (3.8) (with $c=2 / 3$ ) we have

$$
\int_{T}^{2 T}\left|\mathcal{Z}_{2}(\sigma+i t)\right|^{2} d t \ll_{\varepsilon} T^{1+\varepsilon} \quad(5 / 6 \leq \sigma \leq 1),
$$

hence (3.29) follows from (3.23) (with $c=5 / 6$ ) since $5-6 \sigma \leq 8 / 3-2 \sigma$ for $\sigma \geq 7 / 12$. Note that (3.29) sharpens (3.8) (with the best known value $c=2 / 3)$ for $\sigma \geq 5 / 6$.

4. The conjectures on $\mathcal{Z}_{2}(s)$. It seems reasonable that the lower bound (3.20) of Theorem 1 is essentially of the correct order of magnitude. Therefore we formulate the following

Conjecture 1. For any given $\varepsilon>0,1 / 2<\sigma<1$ and $T \gg 1$ we have

$$
\int_{1}^{T}\left|\mathcal{Z}_{2}(\sigma+i t)\right|^{2} d t \ll_{\varepsilon} T^{2-2 \sigma+\varepsilon} .
$$

This conjecture is very strong. It implies the essentially best possible bounds for $E_{2}(T)$ and the eighth moment of $|\zeta(1 / 2+i t)|$. This is contained in

Corollary 2. If Conjecture 1 holds, then

$$
E_{2}(T) \ll_{\varepsilon} T^{1 / 2+\varepsilon} .
$$

Corollary 3. If Conjecture 1 holds, then

$$
\int_{0}^{T}|\zeta(1 / 2+i t)|^{8} d t \ll_{\varepsilon} T^{1+\varepsilon} .
$$

Proof of Corollary 2. From the defining relation (3.2) it is not difficult to obtain (see e.g. $[5,(5.3)])$ that $\left(C_{1}, C_{2}>0,1 \ll H \leq T / 4\right.$ ),

$$
E_{2}(T) \leq C_{1} H^{-1} \int_{T}^{T+H} E_{2}(x) f(x) d x+C_{2} H \log ^{4} T,
$$

where $f(x)(>0)$ is a smooth function supported in $[T, T+H]$ such that $f(x)=1$ for $T+H / 4 \leq x \leq T+3 H / 4$. If we integrate (3.27) from $x=1$ to 
$x=T$ and take into account the defining relation (3.2) of $E_{2}(T)$, we shall obtain

$$
E_{2}(T)=\frac{1}{2 \pi i} \int_{\mathcal{L}} \mathcal{Z}_{2}(s) \frac{T^{s}}{s} d s+O(1) \quad(T>1) .
$$

Then from (4.4) and (4.5) we have $(1 / 2<c<1, T>1)$

$$
E_{2}(T) \leq \frac{C_{1}}{2 \pi i H} \int_{(c)} \frac{\mathcal{Z}_{2}(s)}{s} \int_{T}^{T+H} f(x) x^{s} d x d s+C_{2} H \log ^{4} T,
$$

and we also have an analogous lower bound for $E_{2}(T)$. Since $f^{(r)}(x) \ll_{r} H^{-r}$ it follows that the $s$-integral in (4.6) can be truncated at $|\operatorname{Im} s|=T^{1+\varepsilon} H^{-1}$ with a negligible error, for any $c$ satisfying $1 / 2<c<1$. We take $c=1 / 2+\varepsilon$ and use (4.1), coupled with the Cauchy-Schwarz inequality for integrals, to deduce that

$$
\int_{(1 / 2+\varepsilon)} \frac{\mathcal{Z}_{2}(s)}{s} \int_{T}^{T+H} f(x) x^{s} d x d s \ll_{\varepsilon} H T^{1 / 2+2 \varepsilon},
$$

so that Corollary 2 follows from (4.6) with $H=T^{1 / 2}$.

Proof of Corollary 3. From (3.21) we have

$$
\int_{T}^{2 T}|\zeta(1 / 2+i t)|^{8} d t \ll T^{2 \sigma-1} \int_{0}^{T^{1+\varepsilon}}\left|\mathcal{Z}_{2}(\sigma+i t)\right|^{2} d t \quad(1 / 2<\sigma<1),
$$

so that (4.3) follows from (4.1) and (4.7).

It is plausible that Conjecture 1 is equivalent to Corollary 3 . We can prove something a little weaker. Namely if (4.3) holds, then by the above method one can sharpen (3.29) to

$$
\int_{T}^{2 T}\left|\mathcal{Z}_{2}(\sigma+i t)\right|^{2} d t \ll_{\varepsilon} \begin{cases}T^{4-4 \sigma+\varepsilon} & (1 / 2<\sigma \leq 1), \\ T^{2-2 \sigma+\varepsilon}+T^{-1} & (\sigma \geq 1) .\end{cases}
$$

Conversely, (4.8) (with $\sigma=1-\varepsilon$ ) implies (4.3) by (4.7), so that (4.3) and (4.8) are equivalent.

In view of the discussion on the true order of $I_{\sigma}(T)$, it seems in place to discuss also the problem of the order of $\mathcal{Z}_{2}(\sigma+i t), t \geq t_{0}>0$ and $1 / 2<\sigma<1$. Conjecture 1 says that $\mathcal{Z}_{2}(\sigma+i t)$ is small in mean square. Perhaps it is even also small pointwise, so the following conjecture is now proposed.

Conjecture 2. For any given $\varepsilon>0$ we have

$$
\mathcal{Z}_{2}(\sigma+i t) \ll_{\varepsilon}|t|^{\varepsilon} \quad(\sigma>1 / 2) .
$$


Conjecture 2 is the analogue of the classical Lindelöf hypothesis $\left(\zeta(1 / 2+i t) \ll_{\varepsilon}|t|^{\varepsilon}\right)$ in the equivalent form

$$
\zeta(\sigma+i t) \ll_{\varepsilon}|t|^{\varepsilon} \quad(\sigma>1 / 2) .
$$

Since both $\mathcal{Z}_{2}(s)$ and $\zeta(s)$ take conjugate values at conjugate points, it suffices in (4.9) and (4.10) to assume that $t>0$. Conjecture 2 implies Conjecture 1, which easily follows from

Corollary 4. Conjecture 2 is equivalent to the statement that, for any given $\varepsilon>0$ and $1 / 2<\sigma<1$,

$$
\mathcal{Z}_{2}(\sigma+i t) \ll_{\varepsilon} t^{1 / 2-\sigma+\varepsilon} \quad\left(t \geq t_{0}>0\right) .
$$

Proof. Trivially (4.11) implies (4.9), so we only have to prove that (4.9) implies (4.11). We suppose that $\sigma>1$ and proceed as in the proof of (3.23) to obtain

$$
\begin{aligned}
\mathcal{Z}_{2}(s)= & O\left(\frac{1}{t}\right)+\frac{1}{2 \pi i} \int_{\mathcal{L}} \mathcal{Z}_{2}(w)\left(\int_{T^{1-\varepsilon}}^{\infty}(1-\varrho(x)) x^{w-s-1} d x\right) d w \\
& +\int_{T^{1-\varepsilon}}^{\infty}(1-\varrho(x)) Q_{4}(\log x) x^{-s} d x \quad(T \leq t \leq 2 T) .
\end{aligned}
$$

An integration by parts shows that the last integral above is $\ll t^{\varepsilon-1}$. Since $(1-\varrho(x))^{(r)} \ll_{r} T^{r(\varepsilon-1)}$ for $r=0,1,2, \ldots$, it follows by $r$ integrations by parts that

$$
\int_{T^{1-\varepsilon}}^{\infty}(1-\varrho(x)) x^{w-s-1} d x \ll T^{-A}
$$

for any given $A>0$ if $|\operatorname{Im} w-\operatorname{Im} s|>T^{\varepsilon}$ and $r=r(\varepsilon, A)$ is sufficiently large. Hence (4.12) yields, on replacing $\mathcal{L}$ by the line $\operatorname{Re} w=1 / 2+\delta$,

$$
\begin{aligned}
\mathcal{Z}_{2}(s) & \ll t^{\varepsilon-1}+t^{\varepsilon} \max _{|v-t| \leq t^{\varepsilon}}\left|\mathcal{Z}_{2}(1 / 2+\delta+i v)\right| \int_{T^{1-\varepsilon}}^{\infty} x^{1 / 2+\delta-\sigma-1} d x \\
& \ll t^{\varepsilon-1}+t^{2 \varepsilon+1 / 2-\sigma} \ll t^{2 \varepsilon+1 / 2-\sigma}
\end{aligned}
$$

if $1<\sigma<3 / 2$ and $\delta>0$ is sufficiently small. Finally we use (3.13), (4.9) (with $\sigma=1 / 2+\varepsilon$ ) and (4.13) (with $\sigma=1+\varepsilon$ ) to deduce that

$$
\mathcal{Z}_{2}(s) \ll_{\varepsilon} X^{1 / 2-\sigma} t^{\varepsilon}+X^{1-\sigma} t^{\varepsilon-1 / 2} \ll t^{\varepsilon+1 / 2-\sigma}
$$

for $X=t$. This finishes the proof of Corollary 4 .

In concluding, note that (4.2) and (4.3) do not seem to imply each other. It seems even that the Riemann hypothesis (that all complex zeros of $\zeta(s)$ have real parts equal to $1 / 2$ ) does not imply (4.2). On the other hand, (4.3) is a trivial consequence of the Lindelöf hypothesis (4.2) (which is a consequence of the Riemann hypothesis; see [2] and [17]). 
5. The conjecture on exponential sums with Hecke series. We now pass to a conjecture involving exponential sums with the Hecke series $H_{j}^{3}(1 / 2)$, which will have applications to $\mathcal{Z}_{2}(s)$ and to $\zeta(s)$. The Lindelöf hypothesis $(4.10)$ can be recast (by using the approximate functional equation for $\zeta(s)$ and the Perron inversion formula [2, (A.10)]) in the form involving exponential sums, namely

$$
\sum_{N<n \leq N^{\prime}} n^{-i t} \ll_{\varepsilon} N^{1 / 2} t^{\varepsilon} \quad\left(N<N^{\prime} \leq 2 N \ll t, t>t_{0}>0\right) .
$$

It trivially implies the power moment estimates (see [2], [3] and [17] for a comprehensive account)

$$
\int_{0}^{T}|\zeta(1 / 2+i t)|^{2 k} d t \ll_{\varepsilon, k} T^{1+\varepsilon} \quad(k \in \mathbb{N}),
$$

and in particular the eighth moment (4.3), namely the case $k=4$ of (5.2). The connection with the Hecke series is that both estimates for $E_{2}(T)$ and $\int_{0}^{T}|\zeta(1 / 2+i t)|^{8} d t$ can be made to depend on exponential sums with the Hecke series $H_{j}^{3}(1 / 2)$ (see (5.8) and (5.12)-(5.14)).

One conjectures (this can be thought of as an analogue, in some sense, of the Lindelöf hypothesis) that

$$
H_{j}(1 / 2) \ll_{\varepsilon} \kappa_{j}^{\varepsilon},
$$

and more generally that

$$
H_{j}(1 / 2+i t) \ll_{\varepsilon}\left(\kappa_{j}|t|\right)^{\varepsilon} .
$$

The conjecture (5.3) is, at the present state of knowledge involving Hecke series, certainly out of reach. However, recently the author proved in [7] that

$$
\sum_{K-G \leq \kappa_{j} \leq K+G} \alpha_{j} H_{j}^{3}(1 / 2) \ll_{\varepsilon} G K^{1+\varepsilon}
$$

for

$$
K^{\varepsilon} \leq G \leq K .
$$

In view of the nonnegativity of $H_{j}(1 / 2)$ and $\alpha_{j} \gg_{\varepsilon} \kappa_{j}^{-\varepsilon}$, this result implies that

$$
H_{j}(1 / 2) \ll_{\varepsilon} \kappa_{j}^{1 / 3+\varepsilon} .
$$

Of course, (5.6) is much weaker than the conjectural (5.3), but nevertheless it is the first published improvement over the trivial $H_{j}(1 / 2) \ll \kappa_{j}^{1 / 2}$. The results of (5.4)-(5.6) can be put in the form

$$
\sum_{K-1 \leq \kappa_{j} \leq K+1} \alpha_{j} H_{j}^{3}(1 / 2) \ll_{\varepsilon} K^{1+\varepsilon}
$$


which is essentially best possible. However, when $\alpha_{j} H_{j}^{3}(1 / 2)(\geq 0)$ in (5.7) is weighted by a suitable exponential factor, one expects additional cancellation to take place, just like when instead of the sum

$$
\sum_{N<n \leq N^{\prime}} 1=N^{\prime}-N+O(1) \quad\left(N<N^{\prime} \leq 2 N\right)
$$

we consider the sum in (5.1), which is weighted by the exponential factor $\exp (-i t \log n)$.

In applications an exponential sum with $\alpha_{j} H_{j}^{3}(1 / 2)$ has occurred on at least two important occassions. First, we have (Ivić-Motohashi [10])

$$
\begin{aligned}
E_{2}(T) & \ll \Delta \log ^{c} T \\
+T^{1 / 2} \sup _{\tau \asymp T} \mid & \sum_{\kappa_{j} \leq T \Delta^{-1} \log T} \alpha_{j} H_{j}^{3}(1 / 2) \kappa_{j}^{-3 / 2} \exp \left(i \kappa_{j} \log \frac{\kappa_{j}}{\tau}-\left(\frac{\Delta \kappa_{j}}{T}\right)^{2}\right) \mid
\end{aligned}
$$

provided that $T^{1 / 2} \leq \Delta \leq T^{2 / 3} \log ^{c} T$.

The second application is a bound for the eighth moment of $|\zeta(1 / 2+i t)|$. Note that in $[8]$ it was proved that

$$
\int_{T}^{2 T}|\zeta(1 / 2+i t)|^{8} d t \ll_{\varepsilon} T^{-2} \int_{T^{1 / 2}}^{T^{1+\varepsilon}} t^{2}|\mathcal{G}(1 / 2+i t)|^{2} d t+T^{1+\varepsilon},
$$

where for $1 / 2<a<1$ one has

$$
\begin{aligned}
\mathcal{G}(s) & :=\frac{1}{2 \pi i} \int_{(a)} z_{2}(w) T^{w+s} \frac{U(s, w)}{w} d w \\
U(s, w) & :=\int_{1 / 2}^{5 / 2} \Phi(x) x^{s+w-1} d x \ll \min \left(1, \frac{1}{|s+w|^{A}}\right)
\end{aligned}
$$

for any given $A>0$, where $\Phi(x) \in C^{\infty}$ is a nonnegative function supported in $[1 / 2,5 / 2]$ which equals unity in $[2,2]$. From (5.9) and (5.11) we obtain, by shifting appropriately the line of integration in the expression for $\mathcal{G}(s)$,

$$
\int_{T}^{2 T}|\zeta(1 / 2+i t)|^{8} d t \ll \varepsilon \int_{T^{1 / 2}}^{T^{1+\varepsilon}} t^{2}|I(T, t)|^{2} d t+\Delta(T),
$$

where for the error term $\Delta(T)$ we hope to have $\Delta(T) \ll_{\varepsilon} T^{1+\varepsilon}$, which is by no means easy to establish. In (5.12) we have

$$
I(T, t):=\sum_{t-T^{\varepsilon} \leq \kappa_{j} \leq t+T^{\varepsilon}} \alpha_{j} H_{j}^{3}(1 / 2) \frac{R_{1}\left(-\kappa_{j}\right)}{1 / 2-i \kappa_{j}} \int_{1 / 2}^{5 / 2} \Phi(x)(T x)^{i t-i \kappa_{j}} d x .
$$


The function $R_{1}$ is closely connected to $R$ in (1.2), and we have

$$
\begin{aligned}
R_{1}(y) & :=\sqrt{\frac{\pi}{2}}\left(2^{-i y} \frac{\Gamma(1 / 4-i y / 2)}{\Gamma(1 / 4+i y / 2)}\right)^{3} \Gamma(2 i y) \cosh (\pi y) \\
& \ll(1+|y|)^{-1 / 2} \quad(y \in \mathbb{R}) .
\end{aligned}
$$

If we bound $I(T, t)$ by $(5.7)$ we obtain

$$
I(T, t) \ll t^{-3 / 2} \sum_{t-T^{\varepsilon} \leq \kappa_{j} \leq t+T^{\varepsilon}} \alpha_{j} H_{j}^{3}(1 / 2) \ll t^{\varepsilon-1 / 2} \quad\left(T^{1 / 2} \leq t \leq T^{1+\varepsilon}\right),
$$

and consequently (5.12) gives the worse-than-trivial bound

$$
\int_{0}^{T}|\zeta(1 / 2+i t)|^{8} d t \ll_{\varepsilon} T^{2+\varepsilon} .
$$

The $x$-integral in (5.13) cannot help much because it is practically nonoscillating. One does expect that massive cancellation will be induced by $R_{1}\left(-\kappa_{j}\right)$. From Stirling's formula it follows that

$$
\begin{aligned}
I(T, t)= & O\left(t^{\varepsilon-3 / 2}\right) \\
& +\pi(2 t)^{-3 / 2} \sum_{t-T^{\varepsilon} \leq \kappa_{j} \leq t+T^{\varepsilon}} \alpha_{j} H_{j}^{3}(1 / 2) \exp \left(i \kappa_{j} \log \frac{\kappa_{j}}{4 e}\right) \\
& \times \int_{1 / 2}^{5 / 2} \Phi(x)(T x)^{i t-i \kappa_{j}} d x .
\end{aligned}
$$

The exponential sum involving $\alpha_{j} H_{j}^{3}(1 / 2)$ then becomes essentially the same as the sum in (5.8), if the latter is split into short subsums. Thus it seems reasonable to make the following

Conjecture 3. For $\tau^{\delta} \ll K \ll \tau^{1+\delta}(0<\delta<1)$ we have

$$
\sum_{K-1 \leq \kappa_{j} \leq K+1} \alpha_{j} H_{j}^{3}(1 / 2) \exp \left(i \kappa_{j} \log \frac{\kappa_{j}}{\tau}\right) \ll_{\varepsilon} K^{1 / 2+\varepsilon} .
$$

Note that in (5.16) we are assuming a saving of $\sqrt{K}$ over the known bound (5.7) when there is no exponential factor, which in a sense corresponds to the saving required by the Lindelöf hypothesis (4.10). Very likely (5.16) is, if true, essentially best possible (see (7.6)). Two consequences of (5.16) are the bounds (4.2) and (4.3), similarly to the case when one assumes the conjecture (4.1).

Corollary 5. If Conjecture 3 holds, then (4.2) holds.

Corollary 6. If Conjecture 3 holds, then (4.3) holds. 
Proof of Corollary 5. To obtain (4.2) we use (5.8), splitting the sum into $O(\log T)$ sums over $\left[K, K^{\prime}\right]\left(K<K^{\prime} \leq 2 K\right)$, and removing the monotonic coefficients

$$
\kappa_{j}^{-3 / 2} \exp \left(-\left(\frac{\Delta \kappa_{j}}{T}\right)^{2}\right)
$$

by partial summation. Each of the sums over $\left[K, K^{\prime}\right]$ is further split in $\ll K$ subsums over unit intervals, to which the conjecture (5.16) is applied. The choice $\Delta=T^{1 / 2}$ then gives (4.2).

Proof of Corollary 6. In a similar way we use (5.16) to obtain

$$
I(T, t) \ll_{\varepsilon} t^{1+\varepsilon} \quad\left(T^{1 / 2} \leq t \leq T^{1+\varepsilon}\right),
$$

which easily gives (4.3) by (5.12), provided one can prove rigorously that $\Delta(T) \ll_{\varepsilon} T^{1+\varepsilon}$ in (5.12). This can be achieved by noting that the main contribution to $\Delta(T)$ comes from the bound of the portion of $\mathcal{Z}_{21}(s)$ (see (6.7)) coming from the discrete spectrum at $\sigma=-\varepsilon$, when we shift the line of integration in the relevant part of $(5.10)$ to $a=-\varepsilon$. Then the relevant expression will be an exponential sum with $\alpha_{j} H_{j}^{3}(1 / 2)$ to which (5.16) may be applied. This will lead to $\Delta(T) \ll_{\varepsilon} T^{1+\varepsilon}$.

A possibility to treat the sum in (5.16) is to use Motohashi's transformation formula (see $[15$, Lemma 3.8 and the method of evaluating $\mathcal{C}(K, G)$ on p. 127]) for

$$
\sum_{j=1}^{\infty} \alpha_{j} H_{j}^{3}(1 / 2) h\left(\kappa_{j}\right),
$$

where $h(r)$ is an even function of exponential decay in a suitable horizontal strip satisfying $h(i / 2)=0$. Instead of considering summation over the interval $[K-1, K+1]$, one could consider summation over intervals of the form $[K-G, K+G], K^{\varepsilon} \ll G \ll K^{1-\varepsilon}$, with the idea of choosing $G$ suitably. A natural choice for the function $h$ is

$$
\begin{aligned}
h(r)= & \left(r^{2}+\frac{1}{4}\right) \cos \left(\frac{1}{2} r \log \left(\frac{r}{C T}\right)^{2}\right) \\
& \times\left\{\exp \left(-\left(\frac{r-K}{G}\right)^{2}\right)+\exp \left(-\left(\frac{r+K}{G}\right)^{2}\right)\right\} .
\end{aligned}
$$

This is a difficult problem, and even if some progress with the sum (5.17) could be made, this would not automatically imply any result concerning the sum (5.15), where there is no Gaussian exponential factor.

It may also be remarked that either of the conjectures may be used to derive a mean value result for Dirichlet polynomials. This is 
Corollary 7. If either Conjecture 1,2 or 3 holds, $1 \ll N \ll T^{2}$ and $a(n) \in \mathbb{C}$ is arbitrary, then

$$
\begin{aligned}
\int_{0}^{T}|\zeta(1 / 2+i t)|^{4} \mid & \left.\sum_{N<n \leq N^{\prime} \leq 2 N} a(n) n^{i t}\right|^{2} d t \\
& \ll_{\varepsilon} T^{\varepsilon} \sum_{N<n \leq N^{\prime} \leq 2 N}|a(n)|^{2}\left(T+T^{1 / 2} N\right) .
\end{aligned}
$$

Corollary 7 may be compared to the result of Deshouillers-Iwaniec [1], who had $T+T^{1 / 2} N^{2}+T^{3 / 4} N^{5 / 4}$ as the factor on the right-hand side of (5.18). Assuming the Selberg conjecture that the smallest positive eigenvalue of the non-Euclidean Laplacian for Hecke congruence subgroups is $\geq 1 / 4$, the Deshouillers-Iwaniec proof shows that the term $T^{3 / 4} N^{5 / 4}$ in the above factor may be discarded. This is the limit of the DeshouillersIwaniec method. N. Watt [18] showed that the left-hand side of (5.18) is $\ll_{\varepsilon} T^{\varepsilon}\left(T+T^{1 / 2} N^{2}\right) N \max _{N<n \leq 2 N}\left|a_{n}\right|^{2}$, which improves the DeshouillersIwaniec bound if $a_{n} \ll_{\varepsilon} n^{\varepsilon}$.

6. A new bound for $\mathcal{Z}_{2}(s)$. We have seen that both Conjecture 2 and Conjecture 3 imply the important bounds (4.2) and (4.3) in zeta-function theory. Thus the question naturally arises: is there any connection between Conjecture 2 and Conjecture 3? Does one of them imply the other? They appear to be both of the same level of difficulty, and it will now be shown heuristically that Conjecture 3 implies Conjecture 2. We start from (3.13) and replace the left side of $\mathcal{D}$ by the line $\operatorname{Re} w=-\varepsilon$. Then we expect that the major contribution should come from the poles at $w=1 / 2 \pm i \kappa_{j}-s$. In view of the gamma-factor it then transpires that we obtain the relevant sum

$$
\sum_{\left|\kappa_{j}-t\right| \leq t^{\varepsilon}} \alpha_{j} H_{j}^{3}(1 / 2) X^{1 / 2+i \kappa_{j}-s} R_{1}\left(\kappa_{j}\right) \Gamma\left(1 / 2+i \kappa_{j}-s\right),
$$

where $R_{1}$ is given by (5.14). If we disregard the exponential factor which will come from $R_{1}\left(\kappa_{j}\right)$ and use only (5.7), then from (3.13) and (3.14) (with $X=t)$ we obtain the bound

$$
\mathcal{Z}_{2}(\sigma+i t) \ll_{\varepsilon} t^{1-\sigma+\varepsilon} \quad(1 / 2<\sigma<1),
$$

which improves the bound obtained in [8], where the exponent of $t$ was $2-2 \sigma+\varepsilon$. However, if we use Conjecture 3, then the sum in (6.1) will be $\ll_{\varepsilon} t^{\varepsilon} X^{1 / 2-\sigma}$, and Conjecture 2 follows. We now derive (6.2) rigorously and prove

THEOREM 3. If $s=\sigma+i t$ is well separated from the poles of $\mathcal{Z}_{2}(s)$, then for $0<\sigma<1, t \geq t_{0}>0$ we have

$$
\mathcal{Z}_{2}(\sigma+i t) \ll_{\varepsilon} t^{1-\sigma+\varepsilon} .
$$


Proof. From the bounds [8, (4.13) and (4.29)] we have, under the above hypotheses,

$$
\mathcal{Z}_{2}(\sigma+i t) \ll_{\varepsilon} t^{(1-\sigma) /(1-\xi)+\varepsilon}+\sum_{\left|t-\kappa_{j}\right| \leq t^{\varepsilon}} \alpha_{j} H_{j}^{3}(1 / 2) t^{(\xi-2 \sigma) /(2-2 \xi)+\varepsilon},
$$

provided that

$$
1 / 3 \leq \xi \leq 1 / 2
$$

Take first $\sigma=1 / 2+\delta, \xi=1 / 3$. Then from (6.4) and (5.7) we obtain

$$
\mathcal{Z}_{2}(\sigma+i t) \ll_{\varepsilon} t^{3(1-\sigma) / 2+\varepsilon},
$$

which is weaker than (6.3). The desired bound (6.3) would clearly follow if $\xi=\varepsilon$ is a permissible value in (6.4). To ascertain this fact there are two ways to proceed. Y. Motohashi [13], [15] established the spectral decomposition of the function

$$
\begin{aligned}
\psi(T) & =\psi(T, \xi) \\
& :=\frac{1}{\sqrt{\pi} T^{\xi}} \int_{-\infty}^{\infty}|\zeta(1 / 2+i(T+t))|^{4} \exp \left(-\left(t / T^{\xi}\right)^{2}\right) d t
\end{aligned}
$$

where $0<\xi<1$ is a constant. In [8] this function was used as a substitute for $|\zeta(1 / 2+i T)|^{4}$ when dealing with $\mathcal{Z}_{2}(s)$ for $\sigma \leq 1 / 2$. Namely we put

$$
\begin{aligned}
\mathcal{Z}_{2}(s) & =\int_{1}^{\infty} \psi(T) T^{-s} d T+\int_{1}^{\infty}\left(|\zeta(1 / 2+i T)|^{4}-\psi(T)\right) T^{-s} d T \\
& =\mathcal{Z}_{21}(s)+\mathcal{Z}_{22}(s),
\end{aligned}
$$

say. It turns out that the integral $\mathcal{Z}_{22}(s)$ will converge well, and the main difficulties are inherent in $\mathcal{Z}_{21}(s)$. The key rôle in the spectral decomposition of $\psi(T)$ is played by the function ( $r$ is real)

$$
\begin{aligned}
& \Xi\left(i r ; T, T^{\xi}\right) \\
& =\frac{\Gamma^{2}(1 / 2+i r)}{\Gamma(1+2 i r)} \int_{0}^{\infty}(1+y)^{-1 / 2+i T} y^{-1 / 2+i r} \\
& \quad \times \exp \left(-\frac{1}{4} T^{2 \xi} \log ^{2}(1+y)\right) F(1 / 2+i r, 1 / 2+i r ; 1+2 i r ;-y) d y,
\end{aligned}
$$

where $F$ is the hypergeometric function. For this function Motohashi [15, (5.1.39)-(5.1.41)] obtains an asymptotic formula, where he essentially has the condition (6.5). The first approach is to go carefully through Motohashi's proof of the asymptotic formula for $\Xi\left(\mathrm{ir} ; T, T^{\xi}\right)$, and try to relax the condition (6.5) to $0<\xi \leq 1 / 2$. This can be done, but the analysis is rather long and technical. Here we adopt another approach. It consists in going through the proof of (6.4) in [8], and making appropriate modifications. The condition (6.5) is actually used there in the estimation of the contribution coming 
from the saddle point $z_{0}$, the root of $F(z)=0$, where

$$
F(z)=F(z ; r, T)=-r \log z+T \log \left(1+\frac{z}{T}\right)+2 r \log \left(1+\sqrt{1+\frac{z}{T}}\right),
$$

so that

$$
F^{\prime}(z)=-\frac{r}{z}+\frac{T}{T+z}+\frac{r}{T(\sqrt{1+z / T}+1+z / T)} .
$$

We obtain

$$
\frac{z_{0}}{r}=\left(1+\frac{z_{0}}{T}\right)\left(1+\frac{r / T}{1+\left(1+z_{0} / T\right)^{-1 / 2}}\right)^{-1},
$$

and since it was shown in $[8]$ that

$$
T \geq T(r):=r^{1 /(1-\xi)} \log ^{D} r, \quad|r-t| \leq t^{\varepsilon},
$$

we find by iteration that

$$
\frac{z_{0}}{r}=1+\frac{r}{2 T}+\frac{r^{2}}{8 T^{2}}+O\left(\frac{r^{3}}{T^{3}}\right)
$$

where the $O$-term in (6.9) admits an asymptotic expansion in powers of $r / T$. The crucial term in the contribution of $z_{0}$ is $G\left(z_{0}\right)$, where

$$
\begin{aligned}
G(z):= & i \log \left(1+\frac{z}{T}\right)+\frac{z}{T^{2}} \cdot \frac{1 / 2-i T}{1+z / T} \\
& +\left(1+\sqrt{1+\frac{z}{T}}\right)^{-1} \frac{(1-2 i r) z}{2 T^{2} \sqrt{1+z / T}}-\frac{1}{2} \xi T^{2 \xi-1} \log ^{2}\left(1+\frac{z}{T}\right) \\
& +\frac{1}{2} T^{2 \xi} \log \left(1+\frac{z}{T}\right) \frac{z}{T^{2}},
\end{aligned}
$$

so that

$$
G\left(z_{0}\right)=\frac{i z_{0}}{2 T^{2}}\left(z_{0}-r\right)-\frac{2 i z_{0}^{3}}{3 T^{3}}+O\left(\frac{r}{T^{2}}\right)+O\left(r^{2} T^{2 \xi-3}\right) .
$$

In [8] (cf. page 332, line 7) we estimated trivially $G\left(z_{0}\right)$ as

$$
G\left(z_{0}\right) \ll r^{3} T^{-3}+r T^{-2}+r^{2} T^{2 \xi-3} \ll r T^{-2}+r^{2+\varepsilon} T^{2 \xi-3},
$$

since in view of (6.5) and (6.8) we have

$$
r^{3} T^{-3} \leq r^{2} \log ^{C} r \cdot T^{-2-\xi} \ll_{\varepsilon} r^{2+\varepsilon} T^{2 \xi-3} .
$$

Then by trivial estimation the total contribution coming from the function $\mathcal{Z}_{21}(s)$ is bounded by the second term on the right-hand side of (6.4). However we can deal with the first two terms on the right-hand side of (6.10) as follows. First note that by (6.8) and (6.9),

$$
\frac{i z_{0}}{2 T^{2}}\left(z_{0}-r\right)-\frac{2 i z_{0}^{3}}{3 T^{3}} \asymp \frac{r^{3}}{T^{3}} .
$$


The crucial portion of the integral

$$
X_{r}^{*}(s):=\int_{T(r)}^{\infty} \Xi\left(-i r ; T, T^{\xi}\right) T^{-s} d T
$$

which is to be bounded is of the form

$$
\left(\frac{1}{2}+i r-s\right)^{-1} r^{-1 / 2} \int_{T(r)}^{\infty} K(r, T) e^{i F\left(z_{0}\right)} T^{1 / 2+i r-s} d T,
$$

where $K(r, T) \asymp(r / T)^{3}$ and (6.8) is assumed to hold. A calculation shows that

$$
\frac{\partial F\left(z_{0}\right)}{\partial T} \gg \frac{r^{2}}{T^{2}}
$$

in the relevant range of $r$ and $T$. Write

$$
\int_{T(r)}^{\infty}=\int_{T(r)}^{\infty} K(r, T) T^{1 / 2-\sigma} \cdot e^{i F\left(z_{0}\right)+i(r-t) \log T} d T,
$$

set

$$
H(r, T)=F\left(z_{0}\right)+(r-t) \log T
$$

and observe that, since (6.8) and (6.13) hold,

$$
\frac{\partial H(r, T)}{\partial T} \gg \frac{r^{2}}{T^{2}} \quad\left(\text { for } t \geq T^{1 / 2+\varepsilon}\right) .
$$

Then by the first derivative test (cf. [2, Lemma 2.1]), for $t \geq T^{1 / 2+\varepsilon}$, the total contribution of $r=\kappa_{j}$ does not exceed the second term on the right-hand side of (6.4). In case $t<T^{1 / 2+\varepsilon}$, that is, $T>t^{2-\varepsilon}$, a trivial estimation shows that the contribution of the integral in question is

$$
\begin{aligned}
& \ll_{\varepsilon}|1 / 2+i r-s|^{-1} r^{-1 / 2} \int_{t^{2-\varepsilon}}^{\infty} \frac{r^{3}}{T^{3}} T^{1 / 2-\sigma} d T \\
& \ll_{\varepsilon}|1 / 2+i r-s|^{-1} r^{5 / 2} t^{(2-\varepsilon)(-3 / 2-\sigma)} \ll_{\varepsilon}|1 / 2+i r-s|^{-1} t^{-1 / 2-2 \sigma+\varepsilon} .
\end{aligned}
$$

If $s=\sigma+i t$ is well separated from the poles of $\mathcal{Z}_{2}(s)$, then by (6.8) and (5.7) the total contribution of the portion in question is

$$
\ll_{\varepsilon} \sum_{\left|t-\kappa_{j}\right| \leq t^{\varepsilon}} \alpha_{j} H_{j}^{3}(1 / 2) \cdot t^{-1 / 2-2 \sigma+\varepsilon} \ll_{\varepsilon} t^{1 / 2-2 \sigma+\varepsilon} \ll_{\varepsilon} t^{1-\sigma+\varepsilon} \quad(\sigma \geq-1 / 2),
$$

which is negligible. Since there is now no restriction on $\xi$ except the initial one that $0<\xi \leq 1 / 2$, we obtain (6.3) from (6.4) with $\xi=\varepsilon$, as asserted. We note that one can extend the validity of (6.3) to the half-plane $\sigma>-1 / 2$, since in [8] it was shown that (6.4) holds for

$$
\sigma>\max (2 \xi-1,-1 / 2) \text {. }
$$


In the other direction (6.3) holds at least for $1 \leq \sigma \leq 2$, which follows from (3.24).

We note that the bound

$$
\mathcal{Z}_{2}(1 / 2+\varepsilon+i t) \ll \sqrt{t},
$$

which follows from (6.3), implies the mean square bound (3.15), therefore also (3.16) and (3.19). More generally, if we assume that

$$
\mathcal{Z}_{2}(1 / 2+\varepsilon+i t) \ll t^{\varrho}
$$

with some $0<\varrho \leq 1 / 2$, then from (4.6) we obtain

$$
E_{2}(T) \ll_{\varepsilon} T^{(2 \varrho+1) /(2+2 \varrho)+\varepsilon} .
$$

The bound (6.15) shows that any improvement of (6.3) would have farreaching consequences in zeta-function theory. It also transpires that essentially progress on bounds for $E_{2}(T)$ and the eighth moment of $|\zeta(1 / 2+i t)|$ follows from new bounds for the exponential sums appearing in (5.16).

7. The function $\mathcal{Z}_{k}(s)$. The function $\mathcal{Z}_{2}(s)$ is a special case of the function

$$
\mathcal{Z}_{k}(s):=\int_{1}^{\infty}|\zeta(1 / 2+i x)|^{2 k} x^{-s} d x \quad(s=\sigma+i t ; \sigma, t \in \mathbb{R}, k \in \mathbb{N}),
$$

introduced in [10], where the cases $k=1,2$ were extensively investigated. One of the possible applications of $\mathcal{Z}_{k}(s)$ consists of the following. If $F(s)$ is the Mellin transform of $f(x)$, then by (2.1) one formally obtains, for suitable $c>1$,

$$
\begin{aligned}
\int_{1}^{\infty} f\left(\frac{x}{T}\right)|\zeta(1 / 2+i x)|^{2 k} d x & \\
& =\int_{1}^{\infty} \frac{1}{2 \pi i} \int_{(c)} F(s)\left(\frac{T}{x}\right)^{s} d s|\zeta(1 / 2+i x)|^{2 k} d x \\
& =\frac{1}{2 \pi i} \int_{(c)} F(s) T^{s} \mathcal{Z}_{k}(s) d s
\end{aligned}
$$

If $f(x) \in C^{\infty}$ is a nonnegative function of compact support such that $f(x)=1$ for $1 \leq x \leq 2$, then $F(s)$ is entire of fast decay, and (7.2) (with $c=1+\varepsilon$ ) yields a weak form of the $2 k$ th moment for $|\zeta(1 / 2+i t)|$, namely

$$
\int_{0}^{T}|\zeta(1 / 2+i t)|^{2 k} d t \ll_{k, \varepsilon} T^{1+\varepsilon},
$$

provided that $\mathcal{Z}_{k}(s)$ has analytic continuation to the half-plane $\sigma>1$, where it is regular and of polynomial growth in $|t|$. Conversely, if (7.3) holds, then 
integrating by parts the right-hand side of (7.1) it is seen that $\mathcal{Z}_{k}(s)$ is regular for $\sigma>1$ and in this half-plane satisfies $\mathcal{Z}_{k}(s) \ll_{\sigma}|t|$. Thus the $2 k$ th moment estimate (7.3) has an equivalent formulation in terms of the analytic behaviour of $\mathcal{Z}_{k}(s)$. Moreover (7.3) is equivalent (for example, by Lemma 7.1 of [3]) to the Lindelöf hypothesis. Hence the Lindelöf hypothesis is equivalent to the following statement: given $\varepsilon>0$, for every $k \in \mathbb{N}$ the function $\mathcal{Z}_{k}(s)$ can be analytically continued to the half-plane $\sigma \geq 1+\varepsilon$, where it is of polynomial growth in $|t|$.

In $[8]$ it was shown that

$$
\int_{1}^{T}\left|\mathcal{Z}_{1}(\sigma+i t)\right|^{2} d t \ll_{\varepsilon} T^{2-2 \sigma+\varepsilon} \quad(1 / 2 \leq \sigma \leq 1) .
$$

By arguments analogous to the ones used in the proof of Theorem 1 it can be shown that (7.4) is essentially best possible, namely that the integral in question is $\gg_{\varepsilon} T^{2-2 \sigma-\varepsilon}$. Another, quicker proof of (7.4) follows from the method of proof of Theorem 3. This in fact will yield even

$$
\int_{T}^{2 T}\left|\mathcal{Z}_{1}(\sigma+i t)\right|^{2} d t \ll_{\varepsilon} T^{2-2 \sigma+\varepsilon}+T^{-1} \quad(\sigma \geq 1 / 2) .
$$

The bound (7.4) is the analogue of Conjecture 1 (cf. (4.1)) for $\mathcal{Z}_{1}(s)$. However, the analogue of Conjecture 2 (cf. (4.9)), namely

$$
\mathcal{Z}_{1}(\sigma+i t) \ll_{\varepsilon}|t|^{\varepsilon} \quad(\sigma>1 / 2),
$$

is a difficult problem which is certainly out of reach at present.

One can directly estimate the $2 m$ th moment of $|\zeta(1 / 2+i x)|$ by $\mathcal{Z}_{m}(s)$ as follows. Let $m=k+l, k, l \in \mathbb{N}$. Then by Lemma 1 we obtain, provided $c$ and $d$ are sufficiently large,

$$
\begin{aligned}
\int_{T / 2}^{5 T / 2} \varphi(x) \mid & \left.\zeta(1 / 2+i x)\right|^{2 m} d x \\
& =\frac{1}{2 \pi i} \int_{(c)} \mathcal{Z}_{k}(s) \int_{T / 2}^{5 T / 2} \varphi(x)|\zeta(1 / 2+i x)|^{2 l} x^{s-1} d x d s \\
& =\left(\frac{1}{2 \pi i}\right)^{2} \int_{(c)} \mathcal{Z}_{k}(s) \int_{(d)} \mathcal{Z}_{l}(w) \int_{T / 2}^{5 T / 2} \varphi(x) x^{s+w-2} d x d s d w .
\end{aligned}
$$

Integrating by parts over $x$ sufficiently many times it is seen that the nontrivial contribution in (7.6) comes from $w, s$ satisfying $|v+t| \leq|t|^{\varepsilon}$, where 
$s=\sigma+i t, w=u+i v$. Then from (7.6) we obtain

$$
\int_{T}^{2 T}|\zeta(1 / 2+i x)|^{2 m} d x \ll T^{c+d-1},
$$

provided that $c, d$ are chosen in such a way that the triple integral in (7.6) converges. This procedure shows that mean values of $\mathcal{Z}_{k}(s)$ are relevant not only in the range $\sigma<1$, but in the range $\sigma \geq 1$ as well (see (4.6)). A way to deal with $\mathcal{Z}_{m}(s)$ is to observe that, by Lemma 2 , one has for sufficiently large $c$,

$$
\mathcal{Z}_{m}(s)=\frac{1}{2 \pi i} \int_{(c)} \mathcal{Z}_{l}(w) \mathcal{Z}_{k}(s+1-w) d w
$$

Thus (7.7) is a sort of a recurrent relation that permits one to deduce information on $\mathcal{Z}_{m}(s)$ from $\mathcal{Z}_{k}(s)$ and $\mathcal{Z}_{l}(s)$ if $m=k+l$.

A possible application of (7.6) is to show that if one assumes Conjecture 3 , then it follows that it is essentially optimal. Namely we expect that there exists a constant $C>0$ such that, for given $\delta, \varepsilon>0$, we have

$$
\begin{array}{r|}
\sup _{T^{1-\delta} \leq K \leq T^{1+\delta}}\left|\sum_{K-1 \leq \kappa_{j} \leq K+1} \alpha_{j} H_{j}^{3}(1 / 2) \exp \left(i \kappa_{j} \log \frac{\kappa_{j}}{C T}\right)\right| \\
\gg_{\delta, \varepsilon} T^{1 / 2-\varepsilon} .
\end{array}
$$

This can be shown heuristically as follows. Take $m=4, k=l=2$ in (7.6), so that the left-hand side is $\gg T \log ^{16} T$. From the first identity in (7.6), by using repeated integration by parts and the fact (see Section 3 ) that $Z^{4}(t)$ and its derivatives are bounded in mean by log-powers, it follows that the $s$-integral in (7.6) can be truncated at $|\operatorname{Im} s|=T^{1+\delta}$ with error which is $\ll 1$ when we take $c=1 / 2+\varepsilon$. In the $w$-integral the relevant portion (coming from the discrete spectrum in $\mathcal{Z}_{21}(s)$ in (6.7)) is obtained by taking $a=-\varepsilon$. By using repeated integration by parts in the $x$-integral it transpires that only the terms $\left|t+\kappa_{j}\right| \leq t^{\varepsilon}$ are relevant. Therefore we obtain

$$
\begin{aligned}
T \log ^{16} T \ll & \int_{-T^{1+\delta}}^{T^{1+\delta}}\left|\mathcal{Z}_{2}(1 / 2+\varepsilon+i t)\right| \\
& \times\left|\sum_{\left|\kappa_{j}+t\right| \leq t^{\varepsilon}} \alpha_{j} H_{j}^{3}(1 / 2) R_{1}\left(-\kappa_{j}\right) \int_{T / 2}^{5 T / 2} \varphi(x) x^{\varepsilon-1+t+i \kappa_{j}} d x\right| d t .
\end{aligned}
$$

Conjecture 3, as was seen in Section 6, implies heuristically the truth of Conjecture 2. Thus we may truncate the $t$-integral in (7.9) at $|t|=T^{1-\delta}$ with an error which will be $\ll T$ if $\delta, \varepsilon$ are sufficiently small. Then we use (5.14) 
and Stirling's formula to obtain an asymptotic formula for the function $R_{1}$. Finally we divide the range of summation over the spectrum into $O\left(T^{\varepsilon}\right)$ subsums with the range of summation $K-1 \leq \kappa_{j} \leq K+1$, interchange summation and integration over $x$ and take the suprema over $x$ and $K$ to obtain (7.8) after trivial estimation.

\section{References}

[1] J.-M. Deshouillers and H. Iwaniec, Power mean values of the Riemann zeta-function, Mathematika 29 (1982), 202-212.

[2] A. Ivić, The Riemann Zeta-Function, Wiley, New York, 1985.

[3] - Mean Values of the Riemann Zeta-Function, Tata Inst. Fund. Res. Lectures 82, Bombay, 1991 (distr. by Springer, Berlin).

[4] - On the fourth moment of the Riemann zeta-function, Publ. Inst. Math. (Beograd) (N.S.) 57 (71) (1995), 101-110.

[5] -, The Mellin transform and the Riemann zeta-function, in: Proc. Conference on Elementary and Analytic Number Theory (Vienna, 1996), W. G. Nowak and J. Schoißengeier (eds.), Universität Wien \& Universität für Bodenkultur, Vienna, 1996, 112-127.

[6] - On the error term for the fourth moment of the Riemann zeta-function, J. London Math. Soc. (2) 60 (1999), 21-32.

[7] -, On sums of Hecke series in short intervals, J. Théor. Nombres Bordeaux, to appear.

[8] A. Ivić, M. Jutila and Y. Motohashi, The Mellin transform of powers of the zetafunction, Acta Arith. 95 (2000), 305-342.

[9] A. Ivić and Y. Motohashi, The fourth moment of the Riemann zeta-function, J. Number Theory 51 (1995), 16-45.

[10] - - - The mean square of the error term for the fourth moment of the zetafunction, Proc. London Math. Soc. (3) 66 (1994), 309-329.

[11] A. A. Karacuba and S. M. Voronin, The Riemann Zeta-Function, de Gruyter, Berlin, 1992.

[12] S. Katok and P. Sarnak, Heegner points, cycles and Maass forms, Israel J. Math. 84 (1993), 193-227.

[13] Y. Motohashi, An explicit formula for the fourth power mean of the Riemann zetafunction, Acta Math. 170 (1993), 181-220.

[14] - A relation between the Riemann zeta-function and the hyperbolic Laplacian, Ann. Scuola Norm. Sup. Pisa Cl. Sci. (4) 22 (1995), 299-313.

[15] - Spectral Theory of the Riemann Zeta-Function, Cambridge Univ. Press, Cambridge, 1997.

[16] E. C. Titchmarsh, Introduction to the Theory of Fourier Integrals, Clarendon Press, Oxford, 1948.

[17] - , The Theory of the Riemann Zeta-Function, 2nd ed., Clarendon Press, Oxford, 1986. 
[18] N. Watt, Kloosterman sums and a mean value for Dirichlet polynomials, J. Number Theory 53 (1995), 179-210.

Katedra Matematike RGF-a

Universiteta u Beogradu

Djušina 7, 11000 Beograd

Serbia (Yugoslavia)

E-mail: aleks@ivic.matf.bg.ac.yu

aivic@rgf.rgf.bg.ac.yu

Received on 25.4.2000

and in revised form on 24.2.2001 